\title{
EXACT SOLUTION OF THE NON-LINEAR BOLTZMANN EQUATION FOR MAXWELL MODELS
}

\author{
M.H. ERNST \\ Instituut voor Theoretische Fysica der Rijksuniversiteit, Utrecht, The Netherlands
}

Received 15 November 1978

The exact solution of the Boltzmann equation, obtained recently for Maxwell molecules, holds for various models with arbitrary dimensionality. Furthermore, simple non-linear equations are derived for the ordinary and the Sonine moments, which can be solved subsequentially.

Recently Bobylev [1] and Krook and Wu [2] obtained independently an exact solution of the Boltzmann equation (B.E.) for Maxwell molecules (here referred to as BKW-mode) in the spatially uniform case, in which the distribution function $f(v, t)$ depends only on $|v|=v$. Its possible importance lies in the following conjecture [2]: "an arbitrary initial state tends first to relax to the BKW-mode; the subsequent stage of relaxation is essentially represented by the BKWmode with an appropriate phase". Very recently, Tjon and $\mathrm{Wu}$ [3] gave strong support for the validity of this conjecture by numerically solving a 2-dimensional model for which the BKW-mode is also an exact solution.

It is therefore clearly of importance to find other models, which have the same solution. Here we show that the BKW-mode is an exact solution of the B.E. for arbitrary dimensions $d$ for three classes of Maxwell models (i.e. the collision rate is independent of the velocities of the colliding particles), which all conserve the total number and total energy, and obey the $\mathrm{H}$ theorem. The first class (a) is directly obtained from Maxwell molecules. The second class (b) is a $d$-dimensional version of a 1-dimensional model, proposed by Kac [4]. The third class (c) of models is proposed by Tjon and $\mathrm{Wu}$.

Class (a): For repulsive intermolecular potentials, $r^{-m}$, in $d$-dimensions the differential cross section is $\sigma(g ; \hat{g} \cdot \hat{n})=\alpha(\hat{g} \cdot \hat{n}) g^{-k}$ with $k=2 m^{-1}(d-1)$, where $\boldsymbol{g}=\mathbf{v}-\mathbf{v}_{1}$ and $\boldsymbol{g}^{\prime}=\mathbf{v}^{\prime}-\mathbf{v}_{1}^{\prime}$ are the relative velocities of the colliding particles before and after the collision; the scattering angle $\chi$ is defined through cos $\chi=\hat{g} \cdot \hat{n}$, where $\hat{a}=a / a$ is a unit vector and $\hat{n}=\hat{g}$, and $\alpha(\cos \chi)$ depends only on $\chi$. Hence, for Maxwell molecules the collision rate $g \sigma=\alpha$ is independent of the relative velocity if $m=2(d-1)$, and the B.E. for the spatially uniform case reads

$$
\begin{aligned}
\partial_{t} f & =\int \mathrm{d} \mathbf{v}_{1} \mathrm{~d} \hat{n} \alpha(\hat{g} \cdot \hat{n})\left[f^{\prime} f_{1}^{\prime}-f f_{1}\right] \\
& =\int \mathrm{d} \mathbf{v}_{1} \mathrm{~d} \mathbf{v}^{\prime} \mathrm{d} \mathbf{v}_{1}^{\prime} W\left(\boldsymbol{v} \mathbf{v}_{1} ; \boldsymbol{v}^{\prime} \mathbf{v}_{1}^{\prime}\right)\left[f^{\prime} f_{1}^{\prime}-f f_{1}\right] .
\end{aligned}
$$

The dynamics is given by $\alpha$ and $\mathbf{v}^{\prime}=\boldsymbol{G}+\frac{1}{2} g \hat{n}$ and $\mathbf{v}_{1}^{\prime}=$ $G-\frac{1}{2} g \hat{n}$ with $G=\frac{1}{2}\left(v+v_{1}\right)$, and can also be expressed as a transition probability $W=\alpha g^{1-d} \delta\left(G-G^{\prime}\right) \delta(g-$ $\left.g^{\prime}\right)$. The conservation laws (in appropriate units) are $\int \operatorname{dv}\left(1, \mathbf{v}, v^{2}\right) f(v, t)=(1,0, d)$, and the Maxwellian $f(v, \infty)=(2 \pi)^{-d / 2} \exp \left(-\frac{1}{2} v^{2}\right)$. If we restrict ourselves to pseudo-Maxwell molecules, where the long range part of the potential is cut off, so that that the total classical cross section is finite, then units of time can be chosen such that $\int \mathrm{d} \hat{n} \alpha(\hat{g} \cdot \hat{n})=1$, where $\mathrm{d} \hat{n}$ is a $d$. dimensional solid angle. Further mathematical models are obtained by choosing $\alpha(\cos \chi)$, such as a constant $\alpha$ for isotropic scattering

We start by introducing the characteristic function or Fourier transform $\langle\exp (-\mathrm{i} \boldsymbol{k} \cdot \boldsymbol{v})\rangle=\int \mathrm{d} \boldsymbol{v} \exp (-\mathrm{i} \boldsymbol{k} \cdot \mathbf{v})$ $\times f(v, t) \equiv \Phi\left(\frac{1}{2} k^{2}, t\right)$, which depends only on $|k|=k$, since $f$ is isotropic. In this case the $\hat{v}$-integration can be carried out, yielding $\Phi(x, t)=\left\langle{ }_{0} F_{1}\left(\frac{1}{2} d ;-\frac{1}{2} v^{2} x\right)\right\rangle$, where ${ }_{p} F_{q}$ is a hypergeometric function. The series expansion of ${ }_{0} F_{1}$ generates directly the normalized mo- 
ments [2], $\Phi(x, t)=\Sigma(n !)^{-1}(-x)^{n} M_{n}(t)$, where $n \geqslant 0$ and $M_{n}(t)=N_{n}(t) / N_{n}(\infty)$ with $N_{n}(t)=2^{-n}$ $X\left\langle n^{2 n}\right\rangle$, and $N_{n}(\infty)=\Gamma\left(n+\frac{1}{2} d\right) / \Gamma\left(\frac{1}{2} d\right)$. Through a minor extension of Bobylev's 3-dimensional method we obtain

$\left(\partial_{t}+1\right) \Phi(x)=\int \mathrm{d} \mu g(\mu) \Phi\left(x_{+}\right) \Phi\left(x_{-}\right)$.

Here and in the sequel $-1 \leqslant \mu \leqslant 1$, and $x_{ \pm}=\frac{1}{2}(1 \pm$ $\mu) x$. Furthermore $g(\mu)=b\left(1-\mu^{2}\right)^{p-1} \alpha(\mu)$ with $p=\frac{1}{2}(d-1)$, and specially for isotropic scattering, $g_{0}(\mu, p)=c\left(1-\mu^{2}\right)^{p-1}$. The constants $b$ and $c$ are such that $\int \mathrm{d} \mu g(\mu)=1$. The conservation laws prescribe $\Phi(x, t) \simeq 1-x+\mathrm{O}\left(x^{2}\right)$ for small $x$, and the Maxwellian is $\Phi(x, \infty)=\mathrm{e}^{-x}$.

B.E. (2) has two invariance properties: (i) if $\Phi$ is a solution, so is $\Phi_{s}=\mathrm{e}^{b x} \Phi$ with arbitrary $b$; (ii) B.E. (2) is invariant under the semi-group of similarity transformations $\bar{x}=s^{\lambda} x, \bar{\tau}=s^{\mu} \tau, \bar{\Phi}_{s}=s^{\nu} \Phi_{s}$ with $s>0$ for the values $\mu=\nu=0$ and $\lambda$ (as yet) undetermined.The similarity solution is the special solution, which is itself invariant under this group, i.e. $\Phi_{s}(x, t)=\Phi_{s}\left(s^{\lambda} x ; t+\ln s\right)$ $=\Phi_{s}\left(\mathrm{e}^{-\lambda t} x ; 0\right) \equiv v\left(\mathrm{e}^{-\lambda t} x\right)$. Due to the invariance properties (i) and (ii) (choose $b=1$ ) B.E. (2) allows a similarity solution $\Phi(x, t)=\mathrm{e}^{-x} v\left(\mathrm{e}^{-\lambda t} x\right)$ with boundary conditions $v(x) \simeq 1+\mathrm{O}\left(x^{2}\right)$ for small $x$, consistent with the large $\tau$, and small $x$-behaviour of $\Phi(x, t)$ (at least for $\lambda>0$, as will follow shortly) and satisfying

$v(x)-\lambda x v^{\prime}(x)=\int \mathrm{d} \mu g(\mu) v\left(x_{+}\right) v\left(x_{-}\right)$.

Here $\lambda$ is a non-linear eigenvalue, to be determined from the boundary condition at small $x$. If we take $v(x) \simeq 1+b x^{2}+\ldots$, then $\lambda=\frac{1}{4} \int \mathrm{d} \mu g(\mu)\left(1-\mu^{2}\right)$.

(The only condition imposed by the conservation laws is actually $v(x) \simeq 1+b x^{q}+\ldots$ with $q>1$. This allows for similarity solutions with different eigenvalues [1]). With the above $\lambda$ a special solution of our equation is readily found, i.e. $v(x)=(1-b x) \mathrm{e}^{b x}$ where the constant $b$ may be written as $b=\exp \left(\lambda t_{0}\right)$, and $\Phi(x, t)$ $=(1-a x) \exp [-x(1-a)]$ and $a(t)=\exp \left[-\lambda\left(t-t_{0}\right)\right]$. After inverting the Fourier transform with $x=\frac{1}{2} k^{2}$ one finds in the original velocity variables the exact solution $f(v, t)=\phi_{\alpha}(v)\left[P(t)+Q(t) v^{2}\right]$ with $\phi_{\alpha}(v)=(2 \pi \alpha)^{-d / 2}$ $X \exp \left(-v^{2} / 2 \alpha\right)$ and $P(t)=\frac{1}{2} \alpha^{-1}[(d+2) \alpha-d]$ and $Q(t)=\frac{1}{2} \alpha^{-2}(1-\alpha)$ and $\alpha(t)=1-\exp \left[-\lambda\left(t-t_{0}\right)\right]$ and $t>t_{0}$. This is the $d$-dimensional generalization of the BKW-mode. The parameter $t_{0}$ characterizes the initial distribution, and the choice $\lambda t_{0}>-\log \frac{1}{2}(d+2)$ guar- antees that the initial distribution is positive. For the same reason $b$ is chosen positive. The integral equation (3) can also be viewed [1] as to determine those initial conditions $f(v, 0)$, for which the whole time evolution is given by a mere shift of the parameter $t_{0}$. The nonlinear eigenvalue $\lambda=\frac{1}{2} \mu_{2}$, where $\mu_{n}(n=0,1,2, \ldots)$ are the eigenvalues of the Boltzmann collision operator, linearized around the Maxwellian, i.e. $f(v, t)=f(v, \infty)$ $\times\left[1+h_{n}(v) \exp \left(-\mu_{n} t\right)\right]$. The spectra of these collisions operators are discrete and bounded, i.e. $\mu_{n}=\int \mathrm{d} \mu$ $\times g(\mu)\left[1+\delta_{n 0}-2^{-n}(1+\mu)^{n}-2^{-n}(1-\mu)^{n}\right]$ and the eigenfunctions are the Sonine or associate Laguerre polynomials $h_{n}(v)=L_{n}^{(\alpha)}\left(\frac{1}{2} v^{2}\right)$ with $\alpha=\frac{1}{2}(d-2)$.

There exists a second (Laplace) integral transform method for the non-linear B.E. in the case of isotropic scattering, which is a $d$-dimensional extension of the methods of refs. [2,3]. In this case the equation for $x^{p-1} \Phi(x, t)$ can be transformed into a partial differential equation by taking the Laplace transform, at least when $p=\frac{1}{2}(d-1)$ is a natural number. More generally we consider for arbitrary $p$ the Laplace transform $\int_{0}^{\infty} \mathrm{d} x \mathrm{e}^{-x / \xi} x^{p-1} \Phi(x, t)=\Gamma(p) \xi^{p}\left\langle_{1} F_{1}\left(p ; \frac{1}{2} d ;-\frac{1}{2} v^{2} \xi\right)\right\rangle$ $\equiv \Gamma(p) \xi^{p} G(\xi, t)$, and obtain from B.E. (2),

$$
\left(\partial_{t}+1\right) G(\xi, t)=\int_{0}^{\xi} \mathrm{d} s W_{p}(s ; \xi) G^{2}(s, t) .
$$

The kernel is $w_{p}(x, y)=\Gamma(2 p) \Gamma^{-2}(p)[x(y-x)]^{p-1}$ $\times y^{1-2 p}$. By considering analogous invariance properties, as below (2), we derive an equation for the similarity solution, analogous to (3), and find a special solution for arbitrary $p$.

Before considering the next models we derive equations of motion for the usual moments $M_{n}(t)$, and for the Sonine moments $c_{n}(t)$. As was already known to Maxwell himself, the B.E. for Maxwell molecules gives a rather simple, although non-linear, set of equations for the moments [5], which can be solved sequentially in terms of initial moments $M_{n}(0)$, which are known, once $f(v, 0)$ is given. In our case these equations follow directly by expanding the generating function $\Phi(x, t)$ in B.E. (2) in terms of normalized moments $M_{n}(t)$. The conservation laws give $M_{0}(t)=M_{1}(t)=1$, and for $n \geqslant 2$ we find,

$\dot{M}_{n}+\mu_{n} M_{n}=\sum_{k=1}^{n-1} \mu_{n, k} M_{k} M_{n-k}$ 
with

$\mu_{n, k}=2^{-n}\left(\begin{array}{l}n \\ k\end{array}\right) \int \mathrm{d} \mu g(\mu)(1+\mu)^{k}(1-\mu)^{n-k}$.

In ref. [2] these equations are solved for the model $g(\mu)=g_{0}(\mu ; 1)$ up to $n \leqslant 5$. The Sonine moments, $c_{n}(t)$ with $n=0,1,2, \ldots$ are introduced through the expansion $f(v, t)=f(v, \infty) \Sigma c_{n}(t) L_{n}^{(\alpha)}\left(\frac{1}{2} v^{2}\right)$, so that $c_{n}(t)=n !\left\langle L_{n}^{(\alpha)}\left(\frac{1}{2} v^{2}\right)\right\rangle N_{n}^{-1}(\infty)$. The conservation laws prescribe $c_{0}(t)=1$ and $c_{1}(t)=0$. The Fourier transform of this expansion yields $\Phi(x, t)=\Sigma(n !)^{-1} c_{n}(t)$ $X x^{n} \mathrm{e}^{-x}$. However, since $\mathrm{e}^{x} \Phi(x, t)$ is also a solution of B.E. (2), the Sonine moments satisfy the same moment eq. (5). This property of Maxwell molecules does not seem to have been noticed before. The equations for the Sonine moments in a special 1 -dimensional model (see below) have been obtained before by Kac [4] . It is interesting to notice that the moment $M_{n}(t)$ and $c_{n}(t)$, for $n=2,3$ when calculated from the linearized B.E., are still exact, since eqs. (5) do not involve any non-linear terms. The most important distinction between both types of moments is, that $M_{n}(\infty)=1$ for all $n$, whereas $c_{n}(\infty)=0$ for $n \geqslant 1$.

Class $(b)$ : Kac proposed a simplified 1-dimensional model B.E., whose $d$-dimensional counterpart is obtained from B.E. (1) by replacing $\int \mathrm{d} \hat{n}(\hat{g} \cdot \hat{n})$ with $\int \mathrm{d} \theta \alpha(\theta)$ with $-\pi \leqslant \theta \leqslant \pi$. The (positive) collision rate $\alpha(\theta)$ $=\alpha(-\theta)$ with $\int \mathrm{d} \theta \alpha(\theta)=1$ can be chosen conveniently, and the dynamics is given by a special rotation in a $2 d$ dimensional space, $\mathbf{v}^{\prime}=\mathbf{v} \cos \theta+v_{1} \sin \theta$ and $\mathbf{v}_{1}^{\prime}=-\mathbf{v}$ $X \sin \theta+v_{1} \cos \theta$.

Class (c): These models are proposed by Tjon and $\mathrm{Wu}$ [3], who obtained the BKW-mode as an exact solution for this model in two dimensions. The B.E. is defined in terms of a transition probability $W\left(v v_{1} ; v^{\prime} v_{1}^{\prime}\right)$ $=b\left(v^{2}+v_{1}^{2}\right)^{1-d} \delta\left(v^{2}+v_{1}^{2}-v^{\prime 2}-v_{1}^{2}\right)$. It can be transformed into the usual form where $\int \mathrm{d} \hat{n} \alpha$ in B.E. (1) is replaced by $\int \mathrm{d} \theta \alpha(\theta)$ with $0 \leqslant \theta \leqslant \frac{1}{2} \pi$. The resulting collision rate $\alpha(\theta)=|\sin 2 \theta|^{d-1}$ is again independent of the velocities, and the dynamics is given by $v^{\prime}$ $=\sqrt{v^{2}+v_{1}^{2}} \cos \theta$ and $v_{1}^{\prime}=\sqrt{v^{2}+v_{1}^{2}} \sin \theta$. A convenient representation of the B.E. for class (c) is obtained in terms of the energy distribution function $F(x, t)$ $=\Omega_{d} v^{d-2} f(v, t)$ with $x=\frac{1}{2} v^{2}$ and $\Omega_{d}$ the full $d$. dimensional solid angle,

$\left(\partial_{t}+1\right) F(x)=\int_{x}^{\infty} \mathrm{d} y \int_{0}^{y} \mathrm{~d} z W_{p}(x ; y) F(y-z) F(z)$ with $p=\frac{1}{2} d$. The models (b) and (c) have in common that momentum conservation has been dropped.

The Fourier transform method can be applied directly to B.E. (1) for model (b), and B.E. (2) for $\Phi(x, t)$ follows with $g(\cos \theta)=b\left\{\alpha\left(\frac{1}{2} \theta\right)+\alpha\left(\pi-\frac{1}{2} \theta\right)\right\} \times$ $(\sin \theta)^{-1}$. For model (c) we first derive the moment equations from (6), and obtain from them eq. (2) with $g_{0}\left(\mu ; \frac{1}{2} d\right)$ for the generating function $\Phi(x, t)$ $\left.=\iota_{0} F_{1}\left(\frac{1}{2} d ;-\epsilon x\right)\right\rangle$ with $\epsilon=\frac{1}{2} v^{2}$. Thus, for both classes (b) and (c) all previous results, obtained from the Fourier-transform method, are valid, i.e. the BKWmode as an exact solution; the (non-) linear eigenvalues $\lambda, \mu_{n}$; the eigenfunctions $L_{n}^{(\alpha)}\left(\frac{1}{2} v^{2}\right)$, as well as the moment equations for $M_{n}(t)$ and $c_{n}(t)$. These results apply also to peculiar (b)-models, such as $g(\mu)=\delta(\mu)$. An "isotropic scattering" case in class (b) is obtained by choosing $\alpha(\theta)=|\sin 2 \theta|^{m}$ with $m \geqslant 0$, yielding eq. (2) with $g_{0}\left(\mu ; \frac{1}{2}(m+1)\right)$.

The Laplace transform method can be applied to the "isotropic scattering" cases of class (b), and to the whole class (c). We recover again eq. (4) with $p=\frac{1}{2}(\mathrm{~m}$ +1 ) for class (b), and $p=\frac{1}{2} d$ for class (c). In case $p$ $=\frac{1}{2} d$ (especially class $\left.(\mathrm{c})\right)$ we obtain a nice integral transform, since ${ }_{1} F_{1}\left(\frac{1}{2} d ; \frac{1}{2} d ; z\right)=\mathrm{e}^{z}$, so that $G(\xi, t)=\left\langle\mathrm{e}^{\epsilon \xi}\right\rangle$ is the Laplace transform of the energy distribution function $F(\epsilon, t)$.

Finally, we notice that similarity solutions may also be obtained from (5) for the Sonine moments by putting $c_{n}(t)=b_{n} \mathrm{e}^{-n \lambda t}(n \geqslant 2)$, yielding recurrence relations [1] for the coefficients $b_{n}$.

We would like to thank J. Tjon, T.T. Wu and I. de Schepper for stimulating discussions and M.R. Hoare, who has obtained closely related results, for correspondence.

\section{References}

[1] A.V. Bobylev, Sov. Phys. Dokl. 20 (1976) 820, 822; 21 (1976) 632.

[2] M. Krook and T.T. Wu, Physics of Fluids 20 (1977) 1589.

[3] J. Tjon and T.T. Wu; preprint, Univ. of Utrecht, August 1978.

[4] M. Kac, in: Proc. III Berkeley Symposium on Mathematics, Statistics and Probability (Univ. of California Press, Berkeley, Calif. (1955) p. 171.

[5] C. Truesdell, J. Rat. Mech. Analysis 5 (1976) 55. 